\title{
CONNECTED PHASED ARRAY ANTENNAS FOR ULTRA- WIDE BAND RADAR APPLICATIONS
}

\author{
D. Cavallo, A. Neto, G. Gerini \\ TNO - Defence, Security and Safety \\ Oude Waalsdorperweg 63, 2597 AK, The Hague, The Netherlands \\ E-mail:giampiero.gerini@tno.nl
}

\section{Introduction}

The constantly increasing demand of advanced sensors and communications systems aboard of military platforms (ships, UAVs, aircraft, land vehicles, etc.) requires a high number of antennas, covering a very wide frequency spectrum. An interesting and significant example is given by typical RF functions on board of multifunction frigates. These range from $200 \mathrm{MHz}$ up to $18 \mathrm{GHz}$. The complete band in between these two frequencies is allocated to several functions, including among the others: UHF communication, Electronic warfare Support Measure (ESM), Electronic Counter Measure (ECM), radar, navigation, Identification Friend or Foe (IFF), Joint Tactical Information Distribution System (JTIDS), SHF Satcom etc.

At the same time, the new platform concepts, dictated by an Integrated Topside Design approach, impose a very high level of structural integration of the antenna systems. The overall platform is designed keeping into account many different parameters including Radar Cross Section (RCS), Electromagnetic Interference (EMI), vulnerability etc. All these aspects bring to very stringent constraints for the antennas, in terms of weight, available space and optimal positioning, in order to maintain the requested coverage. Furthermore, this large diversity in systems and technology is also one of the main reasons for the high impact of the costs of such systems on the procurement and maintenance costs of the platforms.

By exploiting and emphasizing the similarities in the requirements of various systems rather than their differences, it is possible to define new scalable architectures. Such systems could be sized for different platforms and adapted to meet different mission needs, therefore allowing an inversion in the cost increasing trends.

From an antenna system point of view, this translates into the necessity of developing antennas easy to be structurally integrated into different platforms and capable of combining more RF functions into the same aperture. In other words: very wide-band active electronically scanned array antennas, preferably in planar printed technology which allows low weight, low profile and low costs. 


\section{Connected arrays}

The most promising array antenna concept, that could meet the very challenging requirements imposed by the above mentioned system architectures, is represented by the so called connected arrays. In the last years, this concept has attracted a growing attention as an alternative to what is, at the moment, the most successful type of wide-band radiators: those belonging to the extended Vivaldi antenna family. These latter types of antennas typically compromise large frequency bandwidth properties with high cross-polarization levels.

Connected arrays present performances in terms of bandwidth, which are comparable to the ones of Vivaldi antennas, but they are much better from the cross-polarization point of view. They are also easier to manufacture, since they can be based on planar printed circuit board technology. Connected arrays typically consist of planar slot or long dipole antennas, periodically fed at Nyquist intervals (see Fig1 a-b.).

Their main characteristic is that the mutual coupling between different feed points is very high, in the order of few $\mathrm{dBs}$. This is the reason why a more suited definition for a connected array is: an array of feeds periodically located in a unique large antenna. This definition is clearly in antithesis with the ones of usual antenna arrays, where the array concept is intended for both the antenna elements and the feeds.

Connected array can be seen as an evolution of the so called self-complementary antennas. These latter are structures whose shape is identical to that of a complementary one, obtained by simply reversing the metallic and non metallic parts (see for example Figure 2).

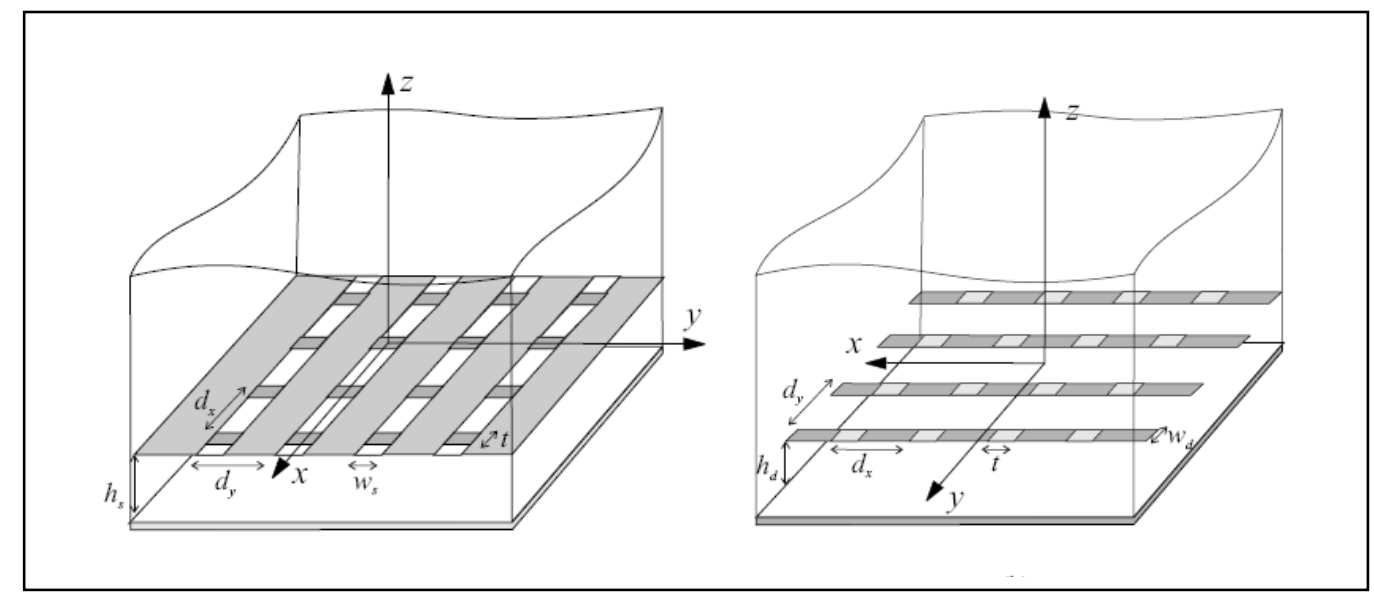

Figure 1: Geometries of 2D connected arrays with backing reflector a) long slot array, b) long dipole array.

The principle and the properties of self complementary antennas were introduced for the first time in the late forties by Y. Mushiake [1]. The most relevant 
characteristic of these antennas is the constant input impedance, independent of the frequency and shape of the structure. The idea of using self-complementary structures for the development of wide band arrays was originally introduced in the late seventies in [2]-[4]. More recently, other works [5], [6] have investigated similar ideas in the form of connected dipoles or patches.

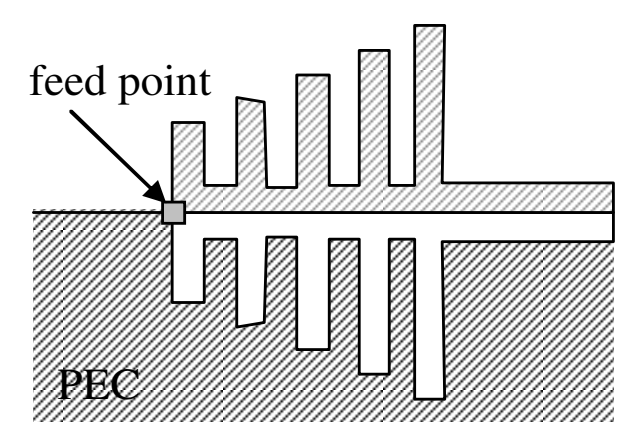

Figure 2: Example of self-complementary antenna structures.

However, only very recently with the works [7]-[10], connected arrays of dipoles and slots have began to be investigated in depth. In particular in [10], the authors presented the development of a wideband compact light weight array operating at UHF for radar and EW applications.

\section{General properties}

The theoretically infinite bandwidth of connected arrays is always achieved at the cost of a $3 \mathrm{~dB}$ loss in gain due to the fact that the antenna radiates equally on the upper and lower half spaces. In reality, the designs of connected arrays can be tuned to achieve almost any wanted impedance bandwidth and the compromise between the bandwidth and the efficiency of the system is typically the driving parameter [9]. If a backing ground plane is used to improve the antenna efficiency, the bandwidth is limited to about $40-45 \%$ with excellent performances in terms of cross-polarization and scanning (up to $\pm 45^{\circ}$ ). This has been proven in the design and development of a hardware demonstrator initiated by TNO. The design has been completed and the results will be presented at the conference. Due to a patent submission procedure, it is not possible at this moment to disclose the details of the structure.

In alternative to a simple backing plane, other solutions characterized by wider bandwidths can be envisaged. For example, an ultra-wide band exceeding 10:1 is feasible at the expense of the efficiency (1 2 dB gain loss), by loading the array with a multi-layer dielectric structure and using absorbing materials in the backside of the antenna. This configuration, in fact, guarantees that most of the power is radiated into the dielectric and the multiple matching layers realize the wide band matching to free space. 
In the following two subsections, the general properties of connected arrays of slots and dipoles will be investigated and compared, providing guidelines for the design of such structures.

\section{Connect array of slots}

The reference connected slot array that we consider is shown in Fig. 1a, together with the pertinent reference system and characterizing parameters. The Green's Function (GF) of an array of long slots periodically fed and in the presence of the backing reflector was derived in [9]. From the GF the input impedance can be expressed explicitly as follows:

$$
Z=\frac{k_{0} \xi_{0} d_{y}}{d_{x}} \sum_{m=-\infty}^{\infty} \frac{\operatorname{sinc}^{2}\left(k_{x m} t / 2\right)}{\left(k_{0}^{2}-k_{x m}^{2}\right) \sum_{n=-\infty}^{\infty} \frac{J_{0}\left(k_{y n} \frac{w_{s}}{2}\right)}{k_{z n}}\left(1-j \operatorname{cotg}\left(k_{z n} h_{s}\right)\right)}
$$

This expression allows some considerations of general validity.

\section{A. Dominant Mode Analysis}

The first consideration is that the impedance presents a clear resonance condition. This condition can be intuited by considering only the first mode, $m=n=0$ in the double summations. Note that this is a realistic hypothesis only when the array is extremely well sampled. In this case the impedance becomes:

$$
Z=\frac{\xi_{0} d_{y}}{d_{x}} \frac{\cos (\theta)}{\left(1-\sin ^{2}(\theta) \cos ^{2}(\phi)\right)\left(1-j \operatorname{cotg}\left(k_{0} \cos (\theta) h_{s}\right)\right)}
$$

Form this expression it is evident that the scanning angle affects not only the amplitude of the impedance, but also the resonance frequency. For larger values of the scanning, e.g. $45^{\circ}$, the electrical length, $k_{0} \cos (\theta) h_{s}$, becomes smaller. This implies that the resonant condition is achieved at a frequency $40 \%$ higher, given that $1 / \cos (\theta) \approx 1$.4. Since the $-10 \mathrm{~dB}$ bandwidth associated to eq.(2) is about $70 \%$ for broadside radiation, even assuming that the BW of the antenna is simply shifted at higher frequency, when scanning, it results that the most highly sampled array would give rise to about $30 \%$ relative bandwidth. An example of this behaviour is shown in Fig. 3, where the reflection coefficient, with respect to a $400 \Omega$ transmission line, of a well sampled array is shown as a function of the frequency for the cases of scanning at broadside and toward $\theta=45^{\circ}$, in the two main planes. Note that similar considerations were already observed for resonant dipole structures from Munk et. al in [11]. 


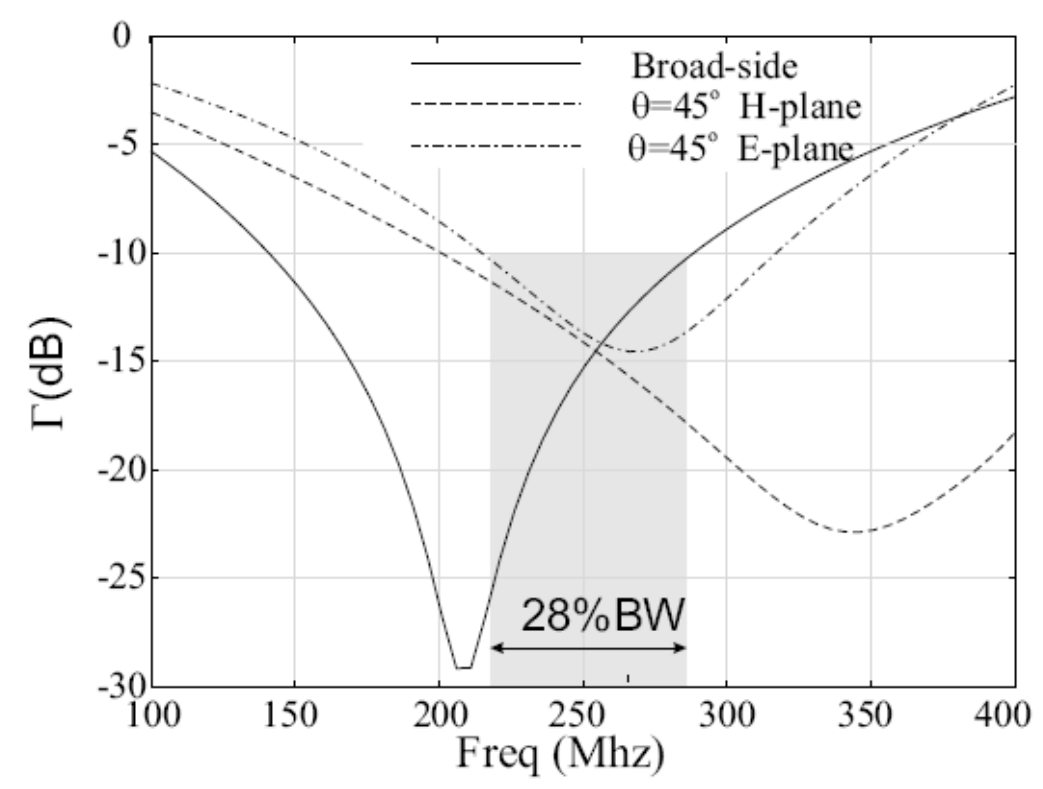

Figure 3: The active reflection coefficient, with respect to a $400 \Omega$, of a slot array characterized by $\mathrm{dx}=\mathrm{dy}=0.4 \mathrm{~m}$., ws $=\mathrm{t}=0.2 \mathrm{~m}$., hs $=0.31 \mathrm{~m}$ scanning at broadside and toward $\theta=45^{\circ}$ in the two main planes.

\section{B. Higher Order Modes}

Higher order modes influence the impedance of a slot connected array in two ways. The modes with very high indices account only for the reactive energy associated to the array. Most of this energy is associated to the series inductance concentrated at the feeding gaps. Since this inductance can be easily tuned out with a matching network, it is not a major parameter in the connected slot array design. The lower order modes, the ones associated to indices $m= \pm 1$ and $n= \pm 1$ will instead affect very negatively the impedance levels for the higher ranges of useful frequencies, especially when the array is scanning to wide angles. This effect can be mitigated by increasing the feeding sampling of the array, therefore retarding the onset of the higher order modes.

Accordingly, in a realistic array design, a compromise must be found between the number of T/R modules in the array and the bandwidth. The array in Fig. 1 is well behaved for scanning in elevation until $45^{\circ}$, because it is sampled with a period of $0.4 \mathrm{~m} .\left(\approx \lambda_{0} / 4\right.$ at $\left.200 \mathrm{MHz}\right)$. When the same array is sampled with a larger period, e.g. $0.55 \mathrm{~m}$., the useful bandwidth is reduced to about $18 \%$, as can be observed in Fig. 4. 


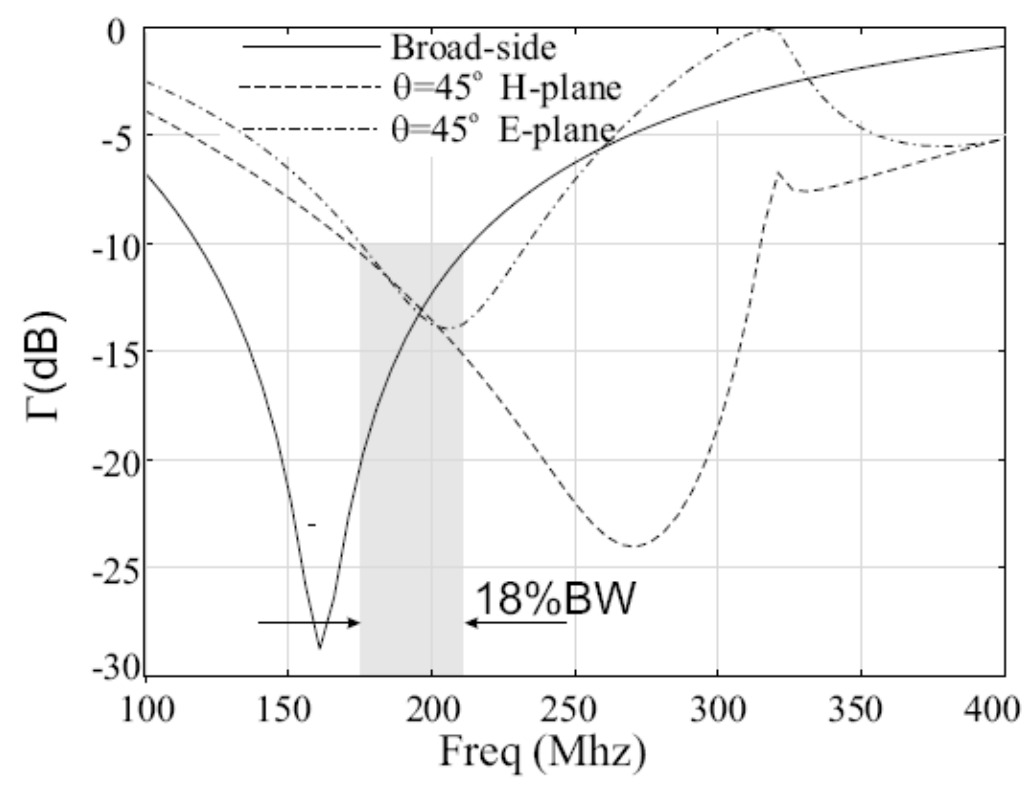

Figure 4: The active reflection coefficient, with respect to a $400 \Omega$, of a slot array characterized by $\mathrm{d}_{\mathrm{x}}=\mathrm{d}_{\mathrm{y}}=0.55 \mathrm{~m}$., $\mathrm{w}_{\mathrm{s}}=\mathrm{t}=0.2 \mathrm{~m}$., $\mathrm{h}_{\mathrm{s}}=0.31 \mathrm{~m}$. scanning at broadside and toward $\theta=45^{\circ}$ in the two main planes.

\section{Leaky Waves}

The only possibility left to the designer to achieve a better matching at higher frequencies is to reduce the distance $h_{s}$ between the ground plane and the antenna aperture plane. However, also this remedy comes at a cost. Fig. 5 shows the real parts of the input impedance of connected slot arrays, over a broad frequency range. Named $\lambda_{c}$ the wavelength at the desired maximum operating frequency, the curves are given for $h_{s}=0.1 \lambda_{c}$ and $h_{s}=0.4 \lambda_{c}$. The remaining array parameters are fixed at $\mathrm{w}_{\mathrm{s}}=0.2 \lambda_{\mathrm{c}}, \mathrm{d}_{\mathrm{x}}=\mathrm{d}_{\mathrm{y}}=0.5 \lambda_{\mathrm{c}}$ and $\mathrm{t}=0.05 \lambda_{\mathrm{c}}$. The curves are also given for different scanning angles (broadside and $45^{\circ}$ in the $\mathrm{E}$ and $\mathrm{H}$ planes).

It is apparent that for smaller heights the resonances are indeed shifted toward higher frequencies. However, smaller heights imply the drastic narrowing of the useful band when scanning in the E plane.

The reason is the compatibility of the parallel plate waveguide structure with a TEM guided mode. This mode, when perturbed by the presence of the periodic slots originates leaky waves which, as they propagate, leak power in radiation, in preferred directions. To investigate the properties of such leaky waves, one can study the propagation of waves launched along the slots. The double spectral summation of the Floquet waves modes in eq.(1) is nested, with the summation of the Floquet waves associated to the direction parallel to the slots $(\mathrm{m})$ being external. Since the dominant mode in such summation is $\mathrm{m}=0$ and 
$\mathrm{k}_{\mathrm{x} 0}=\mathrm{k}_{0} \sin \theta \cos \phi$, it can be useful to investigate the singularities of the spectrum in $\mathrm{k}_{\mathrm{x}}$ when the array is radiating for $\phi=0\left(\mathrm{ky}_{0}=\mathrm{k}_{0} \sin (\theta) \sin (\phi)=0\right)$.

Expected branch point singularities appear in $\pm \mathrm{k}_{0}$. However, also polar singularities emerge from the dispersion equation that is obtained equating to zero the denominator in equation (1). The dispersion equation can be solved approximately using a first order Newton method, with a procedure similar to the one used in [12]. Fig.5 shows the results of the dispersion analysis for $-k_{0}<\operatorname{Re}\left[k_{x}\right]$ $<\mathrm{k}_{0}$ and $-\mathrm{i} \mathrm{k}_{0}<\operatorname{Im}\left[\mathrm{k}_{\mathrm{x}}\right]<\mathrm{jk}$. The continuous curves, as a function of the slots widths, $\mathrm{Ws}$, and parameterized for different heights, $\mathrm{h}_{\mathrm{s}}$, of the antenna with respect to the backing reflector, indicate the location of pole singularities, $\mathrm{k}_{\mathrm{sxp}}$, of the GF. The array is characterized by $\mathrm{d}_{\mathrm{x}}=\mathrm{d}_{\mathrm{y}}=0.5 \lambda_{0}$, with $\lambda_{0}=2 \pi / \mathrm{k}_{0}$.

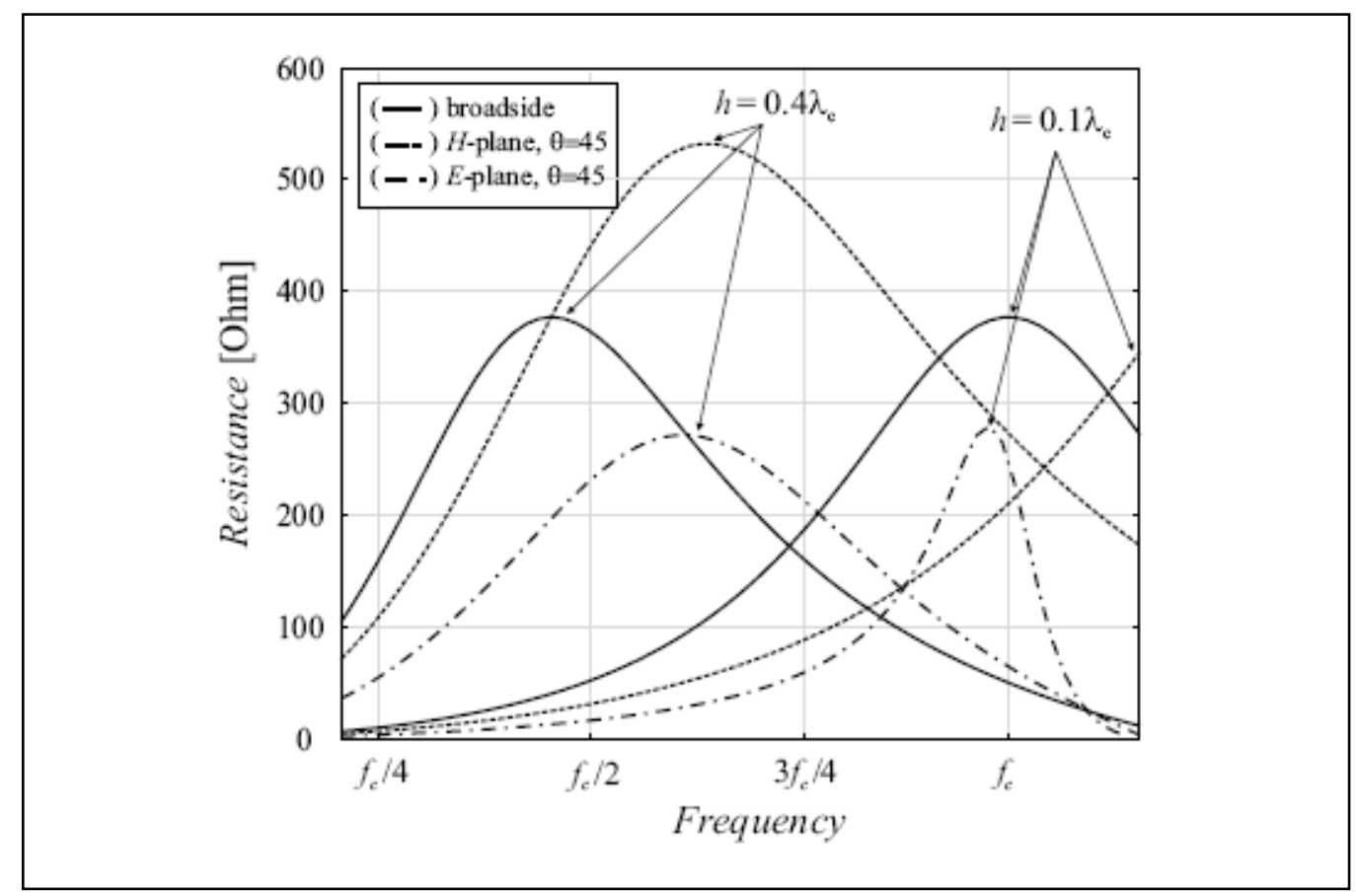

Figure 5: Active impedance of a connected slot array as a function of the frequency for different heights.

The poles represented in Fig. 6 are of the leaky wave type, in the sense that they are characterized by a propagation constant, $\operatorname{Re}\left[\mathrm{k}_{\mathrm{sxp}}\right]$, and by an attenuation constant, $\operatorname{Im}\left[\mathrm{k}_{\mathrm{sxp}}\right]$, this latter associated to radiation losses. It appears that for smaller heights, $h_{s}$, the poles present an increasingly larger imaginary part and, most importantly, a smaller real part, meaning that they can be relevant also for arrays scanning in directions closer to broadside. 


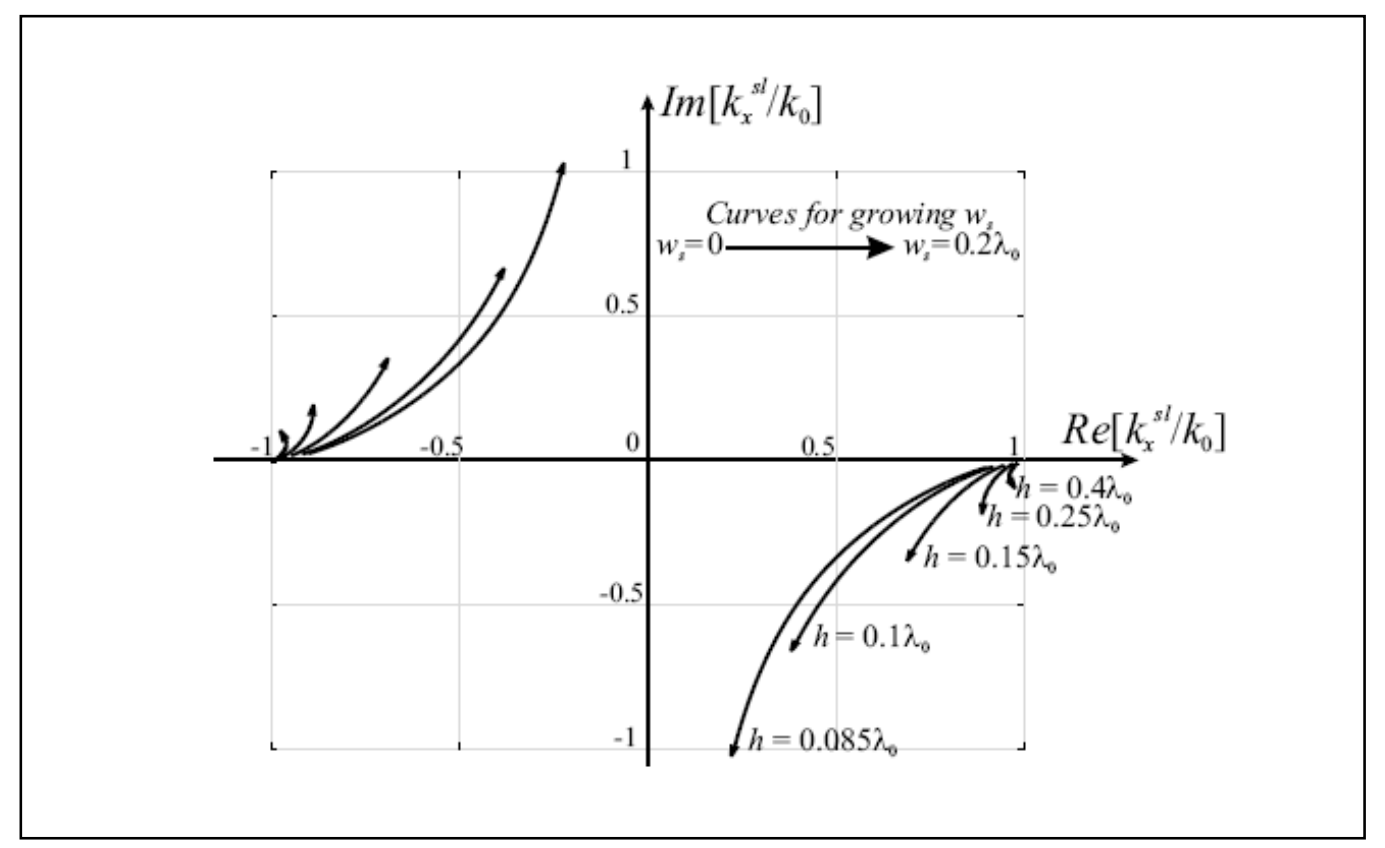

Figure 6: Polar singularities in the complex $\mathrm{k}_{\mathrm{x}}=\mathrm{k}_{0}$ plane when the array is pointing broadside. The dimensions are $\mathrm{d}_{\mathrm{x}}=\mathrm{d}_{\mathrm{y}}=0.5 \lambda_{0} ; \mathrm{t}=0.1 \lambda_{0}$, while $\mathrm{w}_{\mathrm{s}}$ and $\mathrm{h}_{\mathrm{s}}$ are varying.

The intuitive reason for the lower dispersivity, when the backing reflector is further away from the radiating slots, is that the propagating mode of the slot is quasi TEM mode, travelling almost parallel to the slot direction, ksxp. As the backing reflector comes closer, the mode field distributions are much more affected by its presence and thus the propagation becomes more frequency dependent. An increasing width of the slots is also associated to increased dispersivity. In fact, as the slot becomes wider, the field is less tightly bound to the slots and feels the presence of the ground plane. Curves similar to those in Fig. 6 could have been obtained for $\phi \neq 0$, i.e. scanning in planes different from the $\mathrm{E}$ plane. However, equivalent qualitative conclusions would have been obtained. Lower $h_{s}$ are not useful to achieve wide bands for wide scanning angles.

\section{Connect array of dipoles}

The GF of a connected array of dipoles, with backing reflector as in Fig.1b, can be evaluated following an extension of the procedure derived in [9]. The procedure leads to the following expression for the active input admittance at each feed:

$$
Y=\frac{k_{0} d_{y}}{\xi_{0} d_{x}} \sum_{m=-\infty}^{\infty} \frac{\operatorname{sinc}^{2}\left(k_{x m} t / 2\right)}{\left(k_{0}^{2}-k_{x m}^{2}\right) \sum_{n=-\infty}^{\infty} \frac{J_{0}\left(k_{y n} \frac{w_{s}}{2}\right)}{k_{z n}\left(1-j \operatorname{cotg}\left(k_{z n} h_{s}\right)\right)}}
$$


The expression is extremely similar to the one in (1) with the important difference that, independently from the characteristic array parameters, the only pole occurring is located in $\pm k_{0}$ and corresponds to a micro-strip like TEM mode. Such a mode does not have a substantial impact on the antenna scanning performances and there are no effects similar to the ones determined by the leaky waves excited in the slot based structure. Thus, this pole can be essentially neglected also in the design of a scanning array. For what concerns the effects of the scanning angle and of the feed sampling on the bandwidth, we can draw similar conclusions for slot based and dipole based arrays.

The behaviour of the impedance as a function of the (dipole) width is more elaborate than in the slot case. Fig. 7 shows real and imaginary part of such impedance as function of the frequency, with a parametric variation with respect to $\mathrm{w}_{\mathrm{d}}$. The reactive energy localized in the feed gaps is growing as a function of the dipoles width. In circuit terms the characteristic impedance of the micro-strip line, which is slowly varying as a function of the dipoles widths, is in parallel with this feed capacitance. As a consequence, both real and imaginary parts of the impedance vary widely with this geometrical parameter, therefore making the tuning of the structure more complicate and delicate.

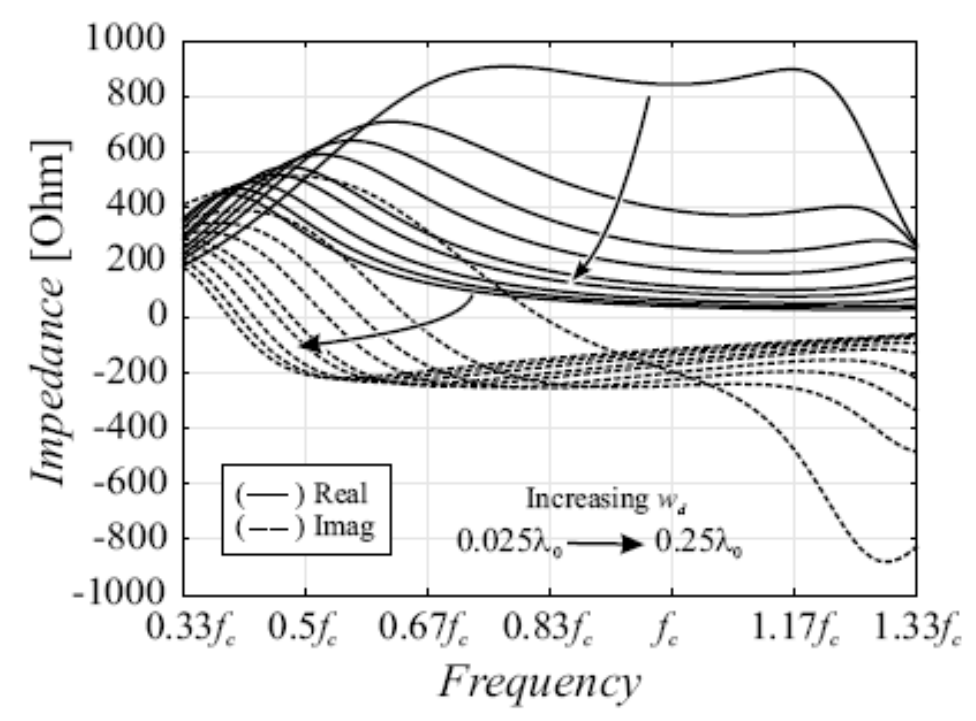

Figure 7: Active impedance of a connected dipole array as a function of the frequency for different widths, given $h_{d}=0.3 \lambda_{c}, t=0.05 \lambda_{c}, d_{x}=d_{y}=0.5 \lambda_{c}$ heights.

\section{Conclusions}

Connected arrays represent one of the most promising array antenna concepts able to meet the very demanding requirements in terms of antenna bandwidth and high polarization purity, imposed by the new generations of radar systems. This array concept can also be applied in other fields like earth based space science (e.g. 
Square Kilometer Array - SKA [13]) or satellite based future missions (e.g. SPICA [14]), as well as for wide-band integrated array antennas for telecommunications. In the present paper, the general properties of arrays of connected slot or dipole antennas have been discussed, providing guidelines for the design of those structures. More details of the hardware demonstrator under development at TNO, which is at the moment under patenting, will be presented at the conference.

\section{References}

[1] Y. Mushiake, "The Input Impedance of a Slit Antenna", Joint Convention Record of Tohoku of IEE and IECE of Japan, 1948, Page(s): 25-26

[2] N. Inagaki, Y. Isogai and Y. Mushiake, "Ichimatsu Moyou Antenna - SelfComplementary Antenna with Periodic Feeding Points", Trans. IECE Japan, Vol. 62-B, Apr. 1979, Page(s): 388-395.

[3] C.E. Baum, "Some Characteristics of Planar Distributed Sources for Radiating Transient Pulses", Sensor and Simulation Note 100, March 12 1970.

[4] C.E. Baum, "Transient Arrays", Ultra-wideband, Short-Pulse Electromagnetics 3, New York: Plenum, 1997.

[5] M.P. Kesler, J.G Maloney, G.S. Smith, "FDTD Analysis of Novel Antenna Array and Substrate Concepts", Proc. ICAP/JINA Conf. AP, April 2000.

[6] P. Friederich et al., "A New Class of Broadband Planar Apertures", Proc. Allerton Antenna Application Symposium, Sept. 2001, Page(s): 561-587.

[7] R.C. Hansen, "Non-Foster and connected planar arrays", Radio Science Vol. 39, 2004, pp. RS4004, 1-14.

[8] J.J. Lee, S. Livingston, R. Koenig, "Wide Band Slot Array Antennas", Proc. IEEE AP Symposium, Vol. 2, Columbus OH, June 2003, Page(s): 452-455.

[9] A. Neto, J.J Lee, "Ultrawide-band Properties of Long Slot Arrays", IEEE Trans. on Antennas and Propagation, Vol. 54, No. 2, Febr. 2006, Page(s): 534-543.

[10] J.J. Lee, S. Livingston, R. Koenig, D. Nagata, L.L. Lai, ”Compact Light Weight UHF Arrays Using Long Slot Apertures", IEEE Trans. on Antennas and Propagation, Vol. 54, No. 7, July 2006, Page(s): 2009 - 2015.

[11] B.A. Munk, "Finite Antenna Arrays and FSS", John Wiley Interscience, 2003.

[12] A. Neto, S. Maci, "Green's Function of an Infinite Slot Printed Between Two Homogeneous Dielectrics. Part I: Magnetic currents", IEEE Transactions on Antennas and Propagation,, Vol. 51, No. 7, July 2003, Page(s): 1572-1581.

[13] D.H. Schaubert, A. van Ardenne, and C. Craeye, "The Square Kilometer Array (SKA) Antenna", IEEE International Symposium on Phased Array Systems and technology, pp. 351-358, October 2003

[14] Core Science Requirements for the European SPICA Instrument, ESIRALREQ-0012, Iss. 0.1. 